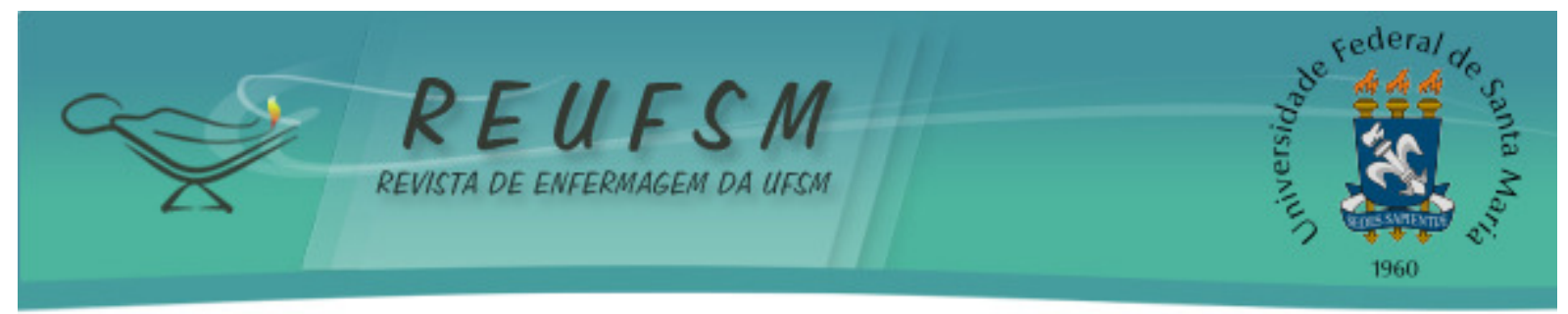

ARTIGO ORIGINAL

\title{
DEMANDAS POR CUIDADOS DE ENFERMAGEM NO DOMICÍLIO POR MULHERES SUBMETIDAS À ONCO-CIRURGIA
}

DEMAND FORNURSING CAREAT HOMEBY WOMEN SUBMITTED TO ONCO SURGERY

DEMANDAS POR ATENCIÓN DE ENFERMERÍA EN EL HOGAR POR MUJERES SOMETIDAS A LA CIRUGÍA ONCOLÓGICA

Doi: $10.5902 / 2179769210497$

Andresa Mendonça de Oliveira ${ }^{1}$ Nathália Silva Gomes ${ }^{2}$

Bibiane Dias Miranda Parreira ${ }^{3}$ Maurícia Brochado Oliveira Soares ${ }^{4}$

Sueli Riul da Silva

RESUMO: Objetivo: identificar demandas por cuidados de enfermagem, pelas portadoras de câncer ginecológico e de mama, em período pós-operatório mediato, no domicílio. Método: quantitativo, descritivo e transversal, realizado com 47 mulheres submetidas a onco-cirurgia no Hospital de Clínicas da Universidade Federal do Triângulo Mineiro. Os dados foram coletados de dezembro de 2008 a julho de 2010 através de visita domiciliar sete dias após a alta hospitalar, sendo utilizado instrumento construído para este estudo. Para análise dos dados utilizou-se estatística descritiva. Resultados: a maioria das clientes $(55,3 \%)$ apresentou dor, $31,9 \%$ alterações de respiração e $29,8 \%$ alterações na ferida cirúrgica. A principal demanda de enfermagem foi orientação. Considerações finais: constatou-se a existência de várias alterações e demandas por parte das mulheres no pós-operatório mediato de oncocirurgia e, muitas destas, podem ser resolvidas na visita domiciliar, pelo enfermeiro.

Descritores: Cuidados de enfermagem; Procedimentos cirúrgicos em ginecologia; Assistência domiciliar.

ABSTRACT: Aim: to identify demands for nursing care for women with breast and gynecological cancer in the mediate post operative period at home. Method: descriptive, cross-sectional, and conducted with 47 women undergoing oncosurgery at the Hospital of the Federal University of Triangulo Mineiro. Data were collected from December 2008 to July 2010, through a home visit seven days after discharge, being built instrument used for this study. Data analysis used descriptive statistics. Results: the majority of clients (55.3\%) presented pain, $31.9 \%$ changes in breathing and $29.8 \%$ changes in the surgical wound. The main demand was nursing orientation. Final thoughts: it was found that there are several changes and demands on the part of women in the mediate postoperative period of oncosurgery and many of these can be resolved in home visits by nurses.

Descriptors: Nursing care; Gynecologic surgical procedures; Home nursing.

\footnotetext{
${ }^{1}$ Graduanda em Enfermagem. Bolsista da FAPEMIG. Universidade Federal do Triângulo Mineiro. andresamendoliver@gamil.com

${ }^{2}$ Enfermeira. Mestre em Atenção à Saúde. Universidade Federal do Triângulo Mineiro. nathaliasilvagomes@hotmail.com

${ }^{3}$ Enfermeira. Mestre em Atenção à Saúde. Universidade Federal Triângulo Mineiro. bibianedias@yahoo.com.br

${ }^{4}$ Enfermeira. Mestre em Atenção à Saúde. Universidade Federal Triângulo Mineiro. mauricia_olive@yahoo.com.br

${ }^{5}$ Enfermeira. Doutora em Enfermagem Fundamental. Universidade Federal do Triângulo Mineiro. sueliriul@terra.com.br
} 


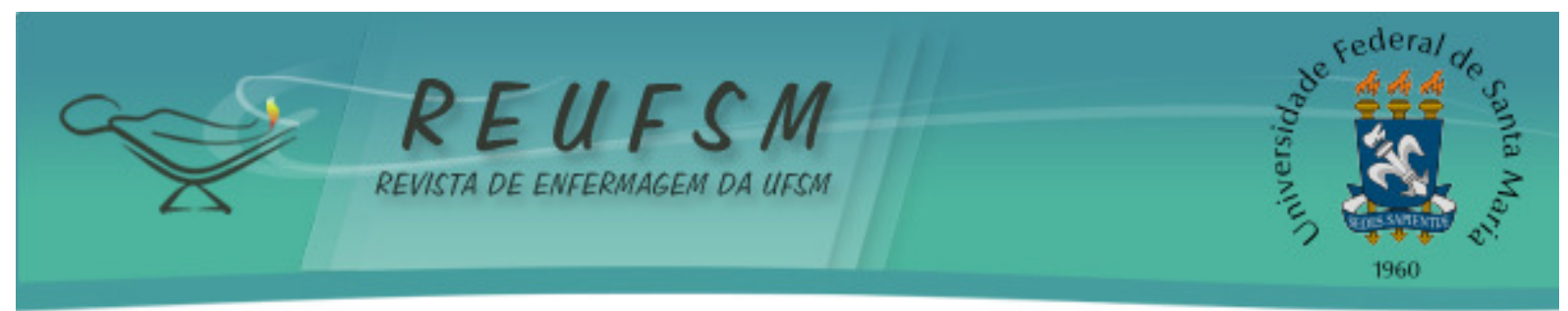

RESUMEN: Objetivo: identificar demandas de atención de enfermería por mujeres con cáncer de mama y ginecológico en el postoperatorio mediato en casa. Método: cuantitativo, descriptivo, transversal, realizado con 47 mujeres que se sometieron a cirugía oncológica en el Hospital de la Universidad Federal del Triángulo Mineiro. Los datos fueron recogidos entre diciembre de 2008 y julio de 2010, través de una visita a la casa siete días después del alta, siendo utilizado instrumento elaborado para este estudio. Para el análisis de datos se utilizó estadística descriptiva. Resultados: la mayoría de las clientes (55,3\%) presentaron dolor, el 31,9\% alteraciones de respiración y el $29,8 \%$ alteraciones en la herida quirúrgica. La demanda principal fue orientación de enfermería. Consideraciones finales: se encontró que hay varios cambios y demandas por parte de las mujeres en el postoperatorio mediato de cirugía onco-ginecológica y muchos de ellos se pueden resolver en las visitas domiciliarias por enfermeras.

Descriptores: Atención de enfermería; Procedimientos quirúrgicos ginecológicos; Atención domiciliaria de salud.

\section{INTRODUÇÃO}

Segundo o Instituto Nacional do Câncer (INCA) as estimativas, no Brasil, para o ano de 2013, apontam para a ocorrência de 518.510 casos novos de câncer (CA). No sexo feminino, com exceção do câncer de pele do tipo não melanoma, o câncer de mama é o mais incidente e o CA de colo uterino ocupa o segundo lugar. ${ }^{1}$

As várias modalidades de tratamento oncológico podem ser divididas em tratamento cirúrgico, radioterapia e tratamento clínico, que engloba imunoterapia, uso de bloqueadores enzimáticos, hormonioterapia, quimioterapia e outros.

O tratamento cirúrgico é a extração de tumores sólidos, assim como de suas regiões adjacentes, visando impedir a propagação regional. ${ }^{2}$ Tem por objetivo a obtenção da cura ou consiste em um método paliativo com a finalidade de reduzir a população de células tumorais facilitando a ação de outras formas de tratamento ou controle de sintomas que comprometam a qualidade de vida do cliente. ${ }^{3}$

Ressaltam-se, aqui, as implicações das onco-cirurgias sobre a qualidade de vida do cliente. Além das complicações inerentes ao CA, os pressupostos da cirurgia oncológica incluem: incisões amplas, ressecções com margens ampliadas dos tumores, remoção de drenagem linfática, entre outros, que geram um comprometimento funcional. De modo geral, o pós-operatório é complicado por amputações com perdas funcionais, imobilizações prolongadas, dificuldade de drenagem linfática, dor intensa, acrescendo as complicações específicas de cada cirurgia. ${ }^{4}$

Somando-se a estes fatores percebe-se que ter um familiar com CA requer uma reorganização do estilo de vida da família, que desenvolvem uma série de ações voltadas à remissão da doença acarretando em mudança de hábitos diários para auxiliar uns aos outros. ${ }^{5}$

Sendo assim, explica-se a necessidade de assistência específica e adequada, bem como acompanhamento a clientes e a seus familiares/cuidadores, sendo esta estendida ao domicílio, local onde há carência de informações e onde se vivencia as alterações referidas, momento em que o enfermeiro tem papel fundamental, contribuindo com práticas de educação em saúde e melhoria da qualidade de vida destes clientes.

Neste contexto, devido às diversas alterações que a sociedade brasileira sofreu no decorrer dos anos (re)surge o modelo de assistência domiciliar, para que promova o desenvolvimento de mudanças sociais e melhorias no sistema de saúde. Este modelo de atenção encontra-se em processo de expansão por todo o Brasil, constituindo-se um novo espaço de trabalho para os profissionais de saúde. Tem como pontos fundamentais o cliente, a família, o contexto domiciliar, o cuidador e a equipe multiprofissional. ${ }^{6}$ 


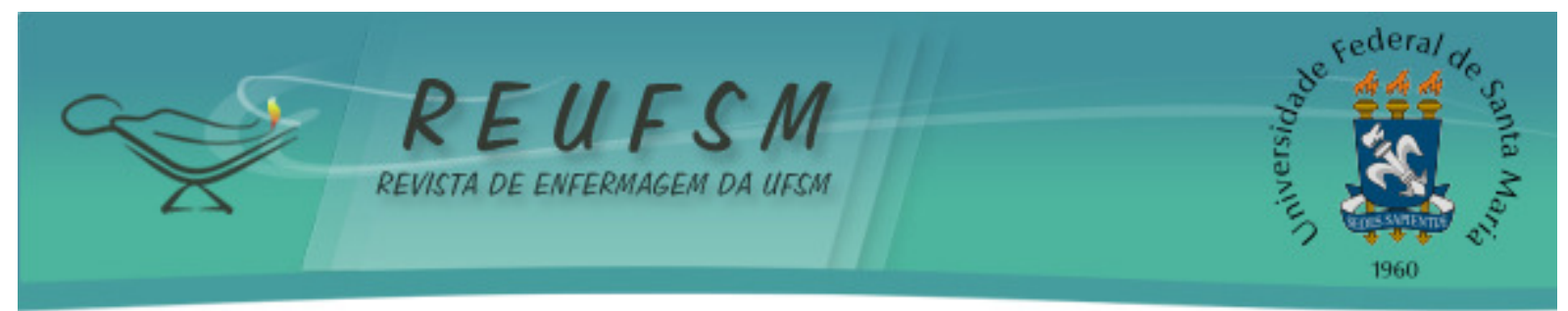

Desta forma, é essencial aprofundar conhecimentos a respeito das necessidades de clientes que se submetem a onco-cirurgia no período mediato, a fim de se obter respostas efetivas e eficazes às demandas dos mesmos, tendo em vista uma proposta terapêutica holística, acompanhando os familiares/cuidadores do cliente devido ao caráter crônico da doença. Nesse contexto, surge o questionamento que instiga e motiva a investigação deste trabalho: quais as demandas por cuidados de enfermagem em mulheres submetidas à oncocirurgia no domicilio?

Portanto, o objetivo do presente estudo foi identificar demandas por cuidados de enfermagem, por portadoras de câncer ginecológico e de mama, em período pósoperatório mediato, no domicílio.

\section{MÉTODO}

Tratou-se de um estudo de caráter quantitativo, descritivo e transversal. Para o seu desenvolvimento foi realizada a identificação das clientes atendidas na Enfermaria de Ginecologia e Obstetrícia do Hospital de Clínicas da Universidade Federal do Triângulo Mineiro (EGO/HC/UFTM) que seriam submetidas à cirurgia onco-ginecológica, mediante acompanhamento da agenda médica de atendimento do Instituto de Pesquisas em Oncoginecologia-IPON, da UFTM.

A visita domiciliar ocorreu dentro da semana seguinte à alta hospitalar onde, após o termo de esclarecimento ter sido lido e o de consentimento assinado, iniciaram-se o levantamento junto às clientes e familiares/cuidadores, sobre as demandas por cuidados no ambiente domiciliar.

Como critérios de inclusão para participação do sujeito na pesquisa foram adotados os seguintes: clientes atendidas no serviço, submetidas à onco-cirurgia, maiores de 18 anos de idade, residentes no município de Uberaba/MG e que concordaram em participar do estudo. Foram excluídas do estudo pacientes com patologia mamária benigna. Foi assegurada a privacidade e o sigilo dos sujeitos participantes da pesquisa, garantidos pela identificação por número.

Os dados foram coletados no período de dezembro de 2008 a julho de 2010, após aprovação do projeto pelo Comitê de Ética em Pesquisa com Seres Humanos da UFTM (Protocolo 1228/2008).

Para a coleta de dados, utilizou-se um instrumento, construído especificamente para este estudo e embasado na literatura científica do tema, dividido em duas partes, a primeira referente ao acompanhamento hospitalar contendo dados de identificação clínica. A segunda parte referente às demandas apresentadas pelas clientes no acompanhamento domiciliar, o qual seguia um roteiro estruturado segundo modelo de coleta de dados de Wanda Horta ${ }^{7}$ acerca das Necessidades Humanas Básicas, proposto por Maslow, sendo os cuidados de enfermagem direcionados de acordo com as demandas individuais, classificadas em Necessidades Psicobiológicas (oxigenação/respiração, circulação, termorregulação, percepção sensorial, integridade tecidual, nutrição/hidratação, eliminação, sono e repouso, atividade física/mobilidade, higiene, sexualidade) Necessidades Psicossociais (segurança, comunicação) e Necessidades Psicoespirituais.

Quanto à análise estatística, realizou-se análise exploratória univariada através da frequência relativa e percentual simples e medidas de posição e de variabilidade.

\section{RESULTADOS E DISCUSSÃO}

Do total de 117 clientes submetidas à onco-cirurgia ginecológica, $50 \quad(42,7 \%)$ atenderam aos critérios de inclusão, destas uma não foi encontrada no domicilio após três 


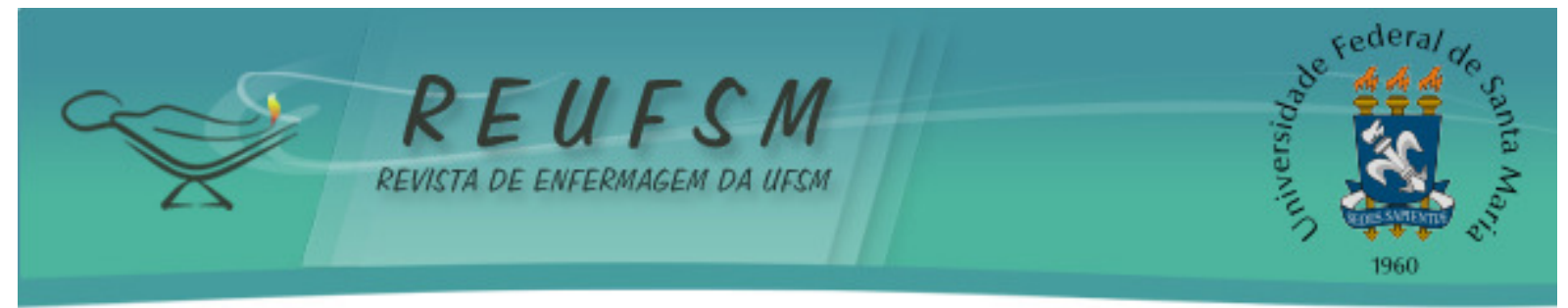

tentativas de busca e, duas recusaram-se a receber a visita domiciliar. Desta forma, a pesquisa foi desenvolvida com 47 clientes.

A idade mínima foi 18 e a máxima 85 anos, sendo a média de 53 anos com desvio padrão de 13,1 e mediana de 51 . Em relação à forma de admissão, 14,9\% foram admitidas pelo Pronto Socorro e 85,1\% faziam acompanhamento pelo Ambulatório Maria da Glória (AMG/HC/UFTM).

No que tange ao diagnóstico, a maior ocorrência foi de CA de mama $(44,7 \%)$, seguido por CA de endométrio (19,1\%), CA de ovário (17\%), CA de colo uterino $(14,9 \%)$, CA de vulva $(2,1 \%)$ e por último, CA de vagina $(2,1 \%)$.

Com relação ao tipo de cirurgia realizada no CA de mama, 10 foram radicais e 11 conservadoras (quadrantectomia), sendo que todas realizaram linfadenectomia axilar.

A escolha da cirurgia depende do estadiamento clínico e do tipo histológico do CA, sendo as cirurgias conservadoras aquelas em que se retira um segmento da mama, com ressecção de margem livre e, a cirurgia radical compreende a mastectomia. ${ }^{2}$ Considerandose que, a taxa de sobrevivência não está relacionada ao tipo de cirurgia, a opção pela cirurgia conservadora está sendo mais utilizada por constituir-se menos mutiladora, ${ }^{8}$ visto a representação da mama para a feminilidade, imagem corporal, sexual e função de amamentação, ${ }^{9}$ sendo sugerido na literatura que o estigma de ter a mama retirada pode levar até ao rompimento da vida sexual do casal. ${ }^{10}$

Além disso, estudo realizado com mulheres com CA de mama aponta que as que possuem melhor qualidade de vida são as que realizaram a quadrantectomia e não necessitaram de reconstrução mamária, seguidas pelo grupo de mastectomizadas que fizeram reconstrução mamária, em contra partida as mastectomizadas que não fizeram reconstrução possuem nível baixo de qualidade de vida, seguidas pelo grupo das mulheres que realizaram quadrantectomia e também não fizeram reconstrução. ${ }^{11}$

Neste sentido, observa-se a necessidade de avaliação criteriosa do tipo da cirurgia a ser realizada e as possibilidades do serviço de saúde oferecer a reconstrução mamária, como formas de melhorar a qualidade de vida destas mulheres.

Concernente aos procedimentos cirúrgicos do CA ginecológico foram realizados: histerectomia total abdominal, salpingooforectomia bilateral, omentectomia e linfadenectomia inguinal em 16 casos, sendo que nestes, em uma cirurgia houve a necessidade de realização de apendicectomia devido aderência ao ovário e em dois casos houve rotura da massa neoplásica; pan-histerectomia em dois casos; ressecção de massa tumoral também em dois casos; histerectomia total abdominal ampliada em três casos; salpingooforectomia em um caso; omentectomia infra-cólica com ooforectomia e vulvectomia radical com linfadenectomia inguinal femoral, ambas também em um caso cada.

Apesar do grande avanço tecnológico no tratamento do CA, este ainda está associado a sentimentos de angústia, sofrimento e impotência frente à doença, nesse sentido o enfermeiro necessita ter um amplo conhecimento científico considerando as alterações emocionais da cliente e de sua família. ${ }^{12}$ Aqui, a visita domiciliar a mulher submetida a onco-cirurgia se torna uma ferramenta importante.

\section{Visita domiciliar}

A visita domiciliar possui caráter pontual, na qual os profissionais observam a realidade do cliente em seu domicílio, incluindo a estrutura física e material, as relações pessoais intra-familiares e realiza as orientações pertinentes. Há também a identificação de problemas e de suas necessidades de saúde. ${ }^{6}$ Desta maneira, esta modalidade de assistência constitui um momento oportuno para que a cliente e/ou seus familiares/cuidadores sanem suas dúvidas, além de proporcionar um atendimento mais 


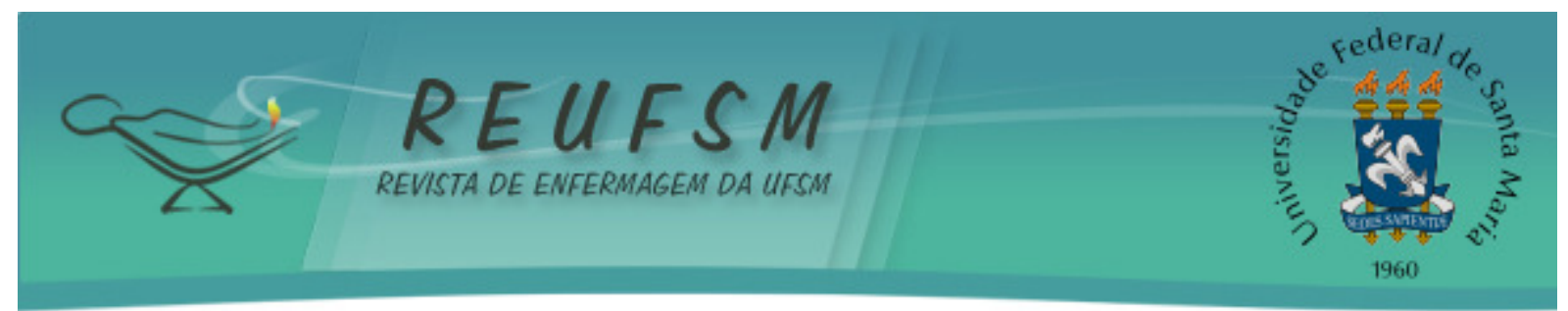

humanizado. 0 que resulta em satisfação e em sensação de acolhimento por parte das clientes, diminuindo as chances de reinternação.

$\mathrm{Na}$ visita domiciliar realizou-se o levantamento das informações junto às clientes e familiares/cuidadores, desenvolveram-se ações de orientação e propostas de soluções para as alterações apresentadas. Os cuidados de enfermagem foram orientados de acordo com as demandas apresentadas, sendo que estas se referiram às necessidades humanas básicas afetadas: alimentação e hidratação, atividade e exercício, higiene, oxigenação, circulação, sono, conforto, eliminação urinária e intestinal. Neste momento, foram abordados os familiares e os cuidadores das clientes, com o intuito de oferecer orientação e apoio, enfatizando a importância dos mesmos neste processo, tendo em vista que a doença não atinge apenas a cliente, mas toda a sua família. ${ }^{5}$

Todas as 47 clientes contavam com o apoio da família/cuidador, que auxiliavam no cuidado dela própria, bem como nos afazeres relacionados ao lar. Como mostra estudo de 2010 realizado em Juiz de Fora/MG, com mulheres que foram submetidas à histerectomia, a maioria das clientes, ao retornarem ao seu domicilio, não se sentiram em condições de realizar as atividades consideradas necessárias à rotina de sua casa e como não podiam fazer certas tarefas, aceitaram ou pediram ajuda de outras pessoas, como familiares, vizinhos, amigos e outros para o desempenho das atividades do lar. ${ }^{13}$

De forma semelhante, em estudo realizado com mulheres submetidas à mastectomia, observou-se que as mesmas, pelas próprias limitações do processo cirúrgico apresentaram impotência para realizar seus afazeres domésticos, mas o suporte familiar desempenhou importante papel apoiando, incentivando e amparando nos momentos mais difíceis vivenciados pelas mulheres com CA. ${ }^{14}$

Neste contexto, também é importante ressaltar a necessidade de orientação quanto ao retorno gradativo às tarefas domésticas e retorno ao seu trabalho o que favorece a alta autoestima. ${ }^{15}$

Após a análise dos dados recolhidos durante a visita domiciliar, dividiu-se as informações em duas categorias: a primeira, referente às necessidades afetadas estão apresentadas no Quadro 1 juntamente com as intervenções de enfermagem baseadas na literatura específica ${ }^{4,16-17}$ e, a segunda, referente às demandas apresentadas pelas mulheres.

\begin{tabular}{|l|l|}
\hline \multicolumn{1}{|c|}{$\begin{array}{c}\text { Necessidades } \\
\text { afetadas }\end{array}$} & \multicolumn{1}{c|}{ Intervenções de enfermagem } \\
\hline Dor - 55,3\% & $\begin{array}{l}\text { - orientar a família e a cliente sobre a dor ou desconforto devido ao } \\
\text { procedimento cirúrgico; } \\
\text { - identificar o uso correto da medicação prescrita pelo médico; } \\
\text { - identificar a intensidade de acordo com a escala numérica de 0 a 10, } \\
\text { localização, frequência, duração e os fatores que melhoram ou pioram a dor; } \\
\text { - manter os medicamentos analgésicos armazenados em local adequado, em } \\
\text { seus frascos originais e fora do alcance de crianças; } \\
\text { - ensinar estratégias adicionais para aliviar a dor e o desconforto como: } \\
\text { distração, relaxamento e terapia com música. }\end{array}$ \\
\hline $\begin{array}{l}\text { Alterações de } \\
\text { respiração }\end{array}$ & $\begin{array}{l}\text { - eliminar os fatores que podem influenciar, como febre e dor; } \\
\text { - evitar grandes esforços; } \\
\text { - promover ambiente seguro e ventilado; } \\
\text { - orientar para procurar o serviço de saúde, se houver agravamento. }\end{array}$ \\
\hline $\begin{array}{l}\text { Alterações na } \\
\text { ferida cirúrgica - } \\
29,8 \%\end{array}$ & $\begin{array}{l}\text { - avaliar o sítio cirúrgico; } \\
\text { - inspecionar a aproximação das bordas e a integridade das suturas; } \\
\text { - verificar presença de sinais flogísticos; } \\
\text { - orientar cuidados como: manter a ferida operatória limpa, lavando-a com }\end{array}$ \\
\hline
\end{tabular}




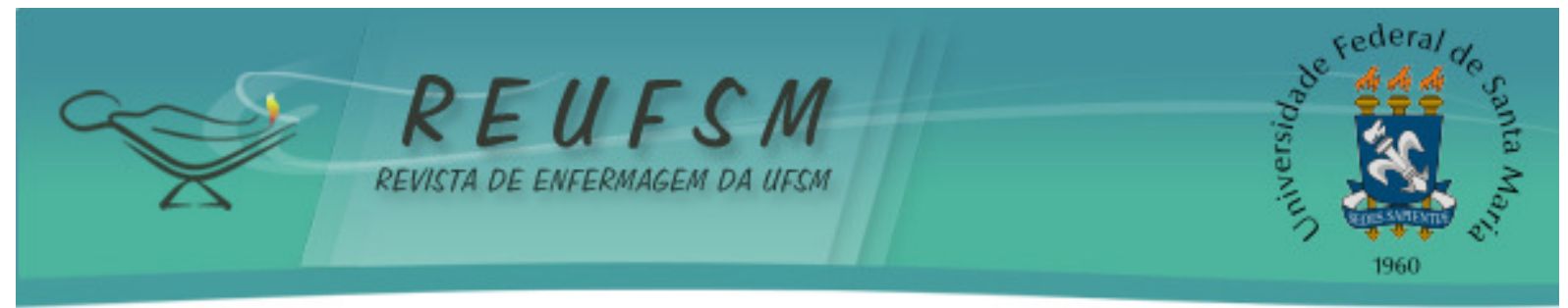

\begin{tabular}{|c|c|}
\hline & $\begin{array}{l}\text { água e sabão neutro, secando-a com toalha limpa, seca e passada, limpando } \\
\text { as regiões laterais da incisão cirúrgica; } \\
\text { - orientar quanto ao debridamento mecânico sendo que a fricção com a } \\
\text { esponja macia deve ser realizada com delicadeza. }\end{array}$ \\
\hline $\begin{array}{l}\text { Hipórexia } \\
25,5 \%\end{array}$ & $\begin{array}{l}\text { - preparar pratos coloridos e variados; } \\
\text { - evitar beber líquidos junto com ou almoço e jantar; } \\
\text { - beber líquidos } 30 \text { minutos antes ou após as refeições; } \\
\text { - fazer refeições leves, de } 3 \text { em } 3 \text { horas. }\end{array}$ \\
\hline $\begin{array}{l}\text { Alteração } \\
\text { eliminação } \\
\text { intestinal } \\
21,3 \%\end{array}$ & $\begin{array}{l}\text { - orientar a beber muito líquido, em torno de oito copos por dia; } \\
\text { - comer alimentos ricos em fibras como os integrais e as frutas frescas tipo } \\
\text { mamão, laranja com bagaço, ameixa, tangerina, uva com casca; } \\
\text { - ingerir verduras como alface, acelga, brócolis, espinafre, couve; } \\
\text { - evitar alimentos como maçã, banana, goiaba, caju, batata, inhame, cará, } \\
\text { creme de arroz, chá preto, refrigerantes e mate; }\end{array}$ \\
\hline Náuseas - 17\% & $\begin{array}{l}\text { - orientar a evitar frituras, alimentos gordurosos e de odor forte; } \\
\text { - fracionar a dieta de } 3 \text { em } 3 \text { horas; } \\
\text { - não ingerir líquidos durante as refeições; } \\
\text { - comer devagar e mastigar bem os alimentos. }\end{array}$ \\
\hline $\begin{array}{l}\text { Alteração de } \\
\text { eliminação } \\
\text { urinária - } 17 \%\end{array}$ & $\begin{array}{l}\text { - ingerir maior quantidade de líquidos; } \\
\text { - observar presença de sangramento; } \\
\text { - procurar o serviço de saúde se houver agravamento. }\end{array}$ \\
\hline $\begin{array}{l}\text { Alteração de } \\
\text { pressão arterial- } \\
14,9 \%\end{array}$ & $\begin{array}{l}\text { - solicitar acompanhamento pelo serviço de saúde; } \\
\text { - orientar sobre os fatores de risco relacionados à hipertensão. }\end{array}$ \\
\hline $\begin{array}{l}\text { Pequena } \\
\text { ingestão de } \\
\text { líquidos }-14,9 \%\end{array}$ & $\begin{array}{l}\text { - observar coloração, turgor, elasticidade, temperatura e umidade cutânea; } \\
\text { - empregar estratégias para encorajar a ingestão de líquidos, como ingerir } \\
\text { pequenos goles com frequência e raspas de gelo; } \\
\text { - realizar registros quanto à ingesta de líquidos }\end{array}$ \\
\hline $\begin{array}{l}\text { Dificuldade de } \\
\text { dormir }-12,8 \%\end{array}$ & $\begin{array}{l}\text { - eliminar ou amenizar os fatores que influenciam o sono utilizando medidas } \\
\text { de conforto; } \\
\text { - orientar quanto à melhor posição para amenizar desconfortos; } \\
\text { - ajudar a cliente a identificar maneiras de eliminar preocupações } \\
\text { estressantes antes de dormir, como ler um livro; } \\
\text { - proporcionar ambiente aconchegante com ventilação adequada e } \\
\text { diminuição de ruídos. }\end{array}$ \\
\hline $\begin{array}{l}\text { Alterações no } \\
\text { dreno de sucção } \\
-6,4 \%\end{array}$ & - procurar o serviço de saúde. \\
\hline $\begin{array}{l}\text { Alteração de } \\
\text { pulso - 4,2\% }\end{array}$ & $\begin{array}{l}\text { - eliminar os fatores que podem influenciar como febre e dor; } \\
\text { - proporcionar ambiente seguro e confortável. }\end{array}$ \\
\hline Febre - 4,2\% & $\begin{array}{l}\text { - orientar a monitoração da temperatura; } \\
\text { - aumentar a ingesta de líquidos; } \\
\text { - orientar banho de aspersão para auxiliar a diminuir a temperatura; } \\
\text { - observar a coloração da pele e presença de tremores; } \\
\text { - orientar a reduzir a frequência de atividades que podem aumentar a } \\
\text { demanda de oxigênio; } \\
\text { - utilizar medicação antitérmica de acordo com a prescrição médica; } \\
\text { - procurar o serviço de saúde caso a febre persista. }\end{array}$ \\
\hline $\begin{array}{l}\text { Perdas } \\
\text { transvaginais } \\
4,2 \%\end{array}$ & $\begin{array}{l}\text { - observar quanto à presença de sangramentos e de odor fétido; } \\
\text { - orientar a procurar o serviço de saúde. }\end{array}$ \\
\hline
\end{tabular}




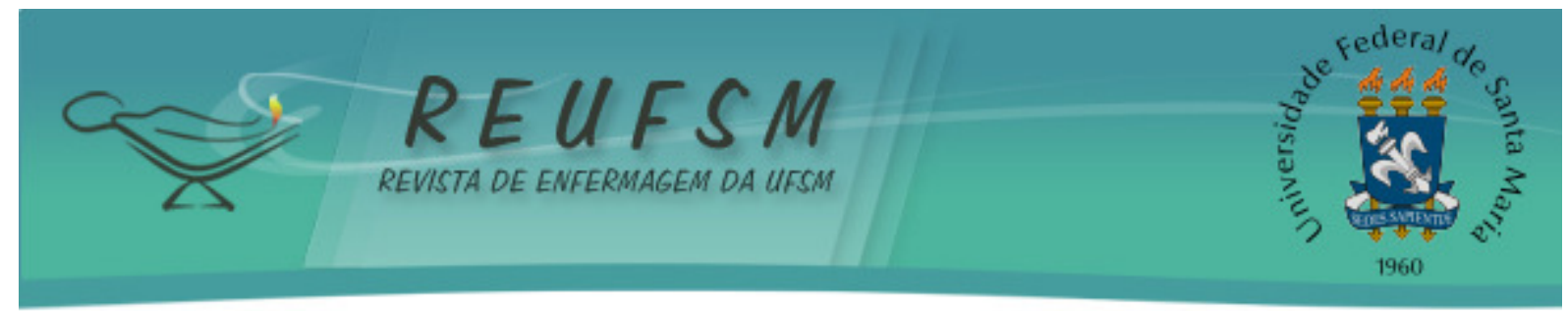

\begin{tabular}{|c|c|}
\hline $\begin{array}{l}\text { Dificuldade } \\
\text { deambular } \\
2,1 \%\end{array}$ & $\begin{array}{l}\text { - estimular a deambulação de forma gradual; } \\
\text { - eliminar possíveis fatores que podem influenciar, como a dor. }\end{array}$ \\
\hline $\begin{array}{l}\text { Edema em MMII } \\
-4,2 \%\end{array}$ & $\begin{array}{l}\text { - manter os membros inferiores elevados; } \\
\text { - controlar ingesta hídrica e de sódio; } \\
\text { - orientar a observar a evolução quanto à intensidade e localização. }\end{array}$ \\
\hline $\begin{array}{l}\text { Restrição ao } \\
\text { leito }-2,1 \%\end{array}$ & $\begin{array}{l}\text { - orientar quanto à mudança de decúbito; } \\
\text { - orientar a utilizar hidratante corporal; } \\
\text { - explicar ao familiar os sinais que antecedem à ferida de decúbito. }\end{array}$ \\
\hline Fraqueza - 2,1\% & $\begin{array}{l}\text { - preferir alimentos nutritivos, ricos em energia e de sua preferência; } \\
\text { - fracionar as refeições e evitar intervalos prolongados entre elas. }\end{array}$ \\
\hline $\begin{array}{l}\text { Reação alérgica } \\
-2,1 \%\end{array}$ & $\begin{array}{l}\text { - identificar o motivo da alergia, neste caso relacionado ao medicamento; } \\
\text { - informar ao médico, que neste caso suspendeu a utilização da medicação; } \\
\text { - orientar quanto ao processo alérgico. }\end{array}$ \\
\hline Calafrios - 2,1\% & - aferir temperatura. \\
\hline $\begin{array}{l}\text { Necessidade de } \\
\text { oxigenoterapia- } \\
2,1 \%\end{array}$ & $\begin{array}{l}\text { - manter a ingesta hídrica adequada; } \\
\text { - orientar quanto aos sinais e sintomas de hipoxemia como dor de cabeça, } \\
\text { sonolência, confusão, palidez, respiração curta e dificuldade de respirar; } \\
\text { - procurar o serviço de saúde caso apresente oscilações incomuns na } \\
\text { frequência respiratória. }\end{array}$ \\
\hline
\end{tabular}

Quadro 1- Descrição das necessidades afetadas e das intervenções de Enfermagem direcionadas às pacientes portadoras de câncer ginecológico, submetidas à onco-cirurgia.

Em relação às demandas, todas as 47 clientes necessitaram de orientação quanto à medicação prescrita pelo médico, 14 pacientes $(29,8 \%)$ precisaram de orientações quanto aos cuidados referentes ao dreno de sucção, demonstrando principalmente ansiedade quanto à sua retirada. A utilização de drenos nestes tipos de cirurgias é comum, visto a extensa dissecção de vasos linfáticos durante o procedimento cirúrgico, o que propicia acúmulo de líquidos e resulta em um espaço morto potencial. ${ }^{9}$

A literatura aponta a importância da visita domiciliar enquanto estratégia de aumentar a adesão ao tratamento, favorecer o autocuidado e oferecer cuidados paliativos ao paciente e sua família, de acordo com suas necessidades e limitações, ${ }^{18}$ tendo em vista que muitos familiares e/ou cuidadores se sentem despreparados para assumir efetivamente o cuidado da mulher submetida a onco-cirurgia. ${ }^{19}$

Durante todo o acompanhamento visou-se incentivar o autocuidado buscando estratégias que amenizem as alterações decorrentes desse processo, proporcionando uma assistência humana de qualidade e holística.

A mulher tem o direito de ser ouvida, opinar sobre suas conveniências, decidir sobre sua própria vida, participar do seu processo de tratamento, inclusive de decidir sobre as modalidades a que será submetida. Ressalta-se que no sistema atual de assistência à saúde, tornam-se evidentes as muitas práticas que desrespeitam o paciente como sujeito e pessoa em seu tratamento. Portanto, é necessário uma proposta em que o modelo assistencial seja traçado pela ética do cuidar. ${ }^{9}$

Os maiores beneficiados da atenção domiciliar à saúde são os clientes e seus familiares, pois o cuidado passa a ser individualizado, humanizado, distante dos riscos 


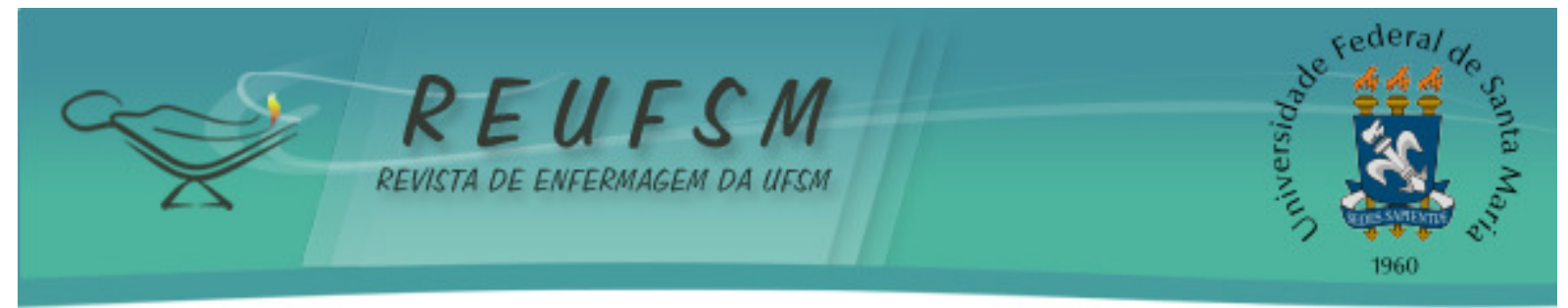

iatrogênicos pertinentes ao contexto hospitalar e ainda conta com a participação do principal cuidador: o familiar ou cuidador. ${ }^{6}$

\section{CONSIDERAÇÕES FINAIS}

Com o intuito de subsidiar uma assistência de enfermagem com qualidade, pôdese observar por meio da análise das categorias de informação extraídas dos dados coletados, a existência de várias alterações e demandas, por parte das mulheres no pósoperatório mediato de onco-cirurgia, destacando-se que muitas destas podem ser resolvidas na visita domiciliar pelo enfermeiro.

Com a realização da cirurgia oncológica várias modificações ocorrem na vida da mulher, sendo de suma importância que o enfermeiro desenvolva no domicílio o papel de orientador, sanando as dúvidas existentes, proporcionando conforto e segurança à cliente e familiares/cuidadores. Destaca-se que as relações afetivas são importantes para o bem-estar físico e psicológico do paciente, os familiares servem de apoio e colaboram com os cuidados relacionados à cliente e ao seu domicílio e são pessoas indispensáveis durante e após o tratamento.

Tendo em vista o caráter crônico da doença é imprescindível obter conhecimentos relativos às necessidades das pacientes, a fim de se dar respostas sempre mais efetivas e eficazes às demandas. A assistência deve ser baseada em conhecimentos científicos sem esquecer a humanização, peça chave para o estabelecimento do vínculo entre profissional e clientes, tendo em vista uma proposta terapêutica holística.

\section{REFERÊNCIAS}

1. Ministério da Saúde (BR). Instituto Nacional do Câncer. Estimativa 2012: incidência de câncer no Brasil [Internet]. Rio de Janeiro: INCA; 2011. [acesso em 2013 ago15]. Disponível em: http://www.inca.gov.br.

2. Instituto Nacional do Câncer. Ações de enfermagem para o controle do câncer: uma proposta de integração ensino-serviço. $3^{\mathrm{a}}$ ed. Rio de Janeiro: INCA; 2008.

3. Bonassa EMA, Gato IR. Terapêutica oncológica para enfermeiros e farmacêuticos. $4^{\mathrm{a}}$ ed. São Paulo: Atheneu; 2012.

4. Smeltzer SC, Bare BG, Hinkle JL, Cheever KH. Brunner \& Suddarth: tratado de enfermagem médico-cirúrgica. 12 ${ }^{\mathrm{a}}$ ed. Rio de Janeiro: Guanabara Koogan; 2012.

5. Visoná $F$, Prevedello $M$, Souza EN. Câncer na família: percepções de familiares. Rev Enferm UFSM [Internet]. 2012 jan/abr [acesso em 2013 jul 20];2(1):145-55. Disponível em: http://cascavel.ufsm.br/revistas/ojs-2.2.2/index.php/reufsm/article/view/3943/3148.

6. Lacerda MR, Giacomozzi CM, Oliniski SR, Truppel TC. Atenção à saúde no domicílio: modalidades que fundamentam sua prática. Saúde Soc [Internet]. 2006;15(2):88-95.

7. Horta WA. Processo de enfermagem. São Paulo: EPU; 1979.

8. Veiga DF, Campos FSM, Ribeiro LM, Archangelo Junior I, Veiga Filho J, Juliano Y, et al. Mastectomy versus conservative surgical treatment: the impact on the quality of life of women with breast cancer. Rev Bras Saúde Matern Infant. 2010 Jan-Mar;10(1):51-7.

9. Arantes SL, Mamede MV. A participação das mulheres com câncer de mama na escolha do tratamento: um direito a ser conquistado. Rev Latinoam Enferm. 2003;11(1):49-58. 


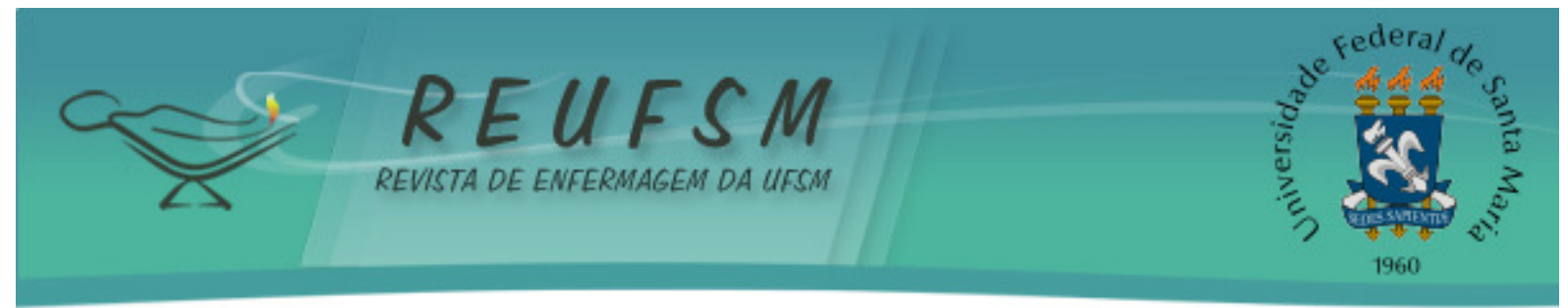

10. Silva TBC, Santos MCL, Almeida AM, Fernandes AFC. Percepção dos cônjuges de mulheres mastectomizadas com relação à convivência pós-cirurgia. Rev Esc Enferm USP. 2010;44(1):113-9.

11. Simeão SFAP, Landro ICR, De Conti MHS, Gatti MAN, Delgallo WD, Vitta A. Qualidade de vida em grupos de mulheres acometidas de câncer de mama. Ciênc Saúde Coletiva. 2013;18(3):779-88.

12. Rubini AMS, Santos JLG, Erdmann AL, Rosa LM. Discursos de mulheres com câncer cervical em tratamento braquiterápico: subsídios para o cuidado de enfermagem. Rev Enferm UFSM [Internet]. 2012 set/dez [acesso em 2013 jul 15];2(3):601-9. Disponível em: http://cascavel.ufsm.br/revistas/ojs-2.2.2/index.php/reufsm/article/view/6865.

13. Salimena AMO, Souza IEO. Cotidiano da mulher pós-histerectomia à luz do pensamento de Heidegger. Rev Bras Enferm. 2010;63(2):196-202.

14. Mistura C, Carvalho MFAA, Santos VEP. Mulheres mastectomizadas: vivências frente ao câncer de mama. Rev Enferm UFSM [Internet]. 2011 maio/ago [acesso em 2013 jul 10];1(3):351-9. Disponível em: http://cascavel.ufsm.br/revistas/ojs2.2.2/index. php/reufsm/article/view/2943.

15. Gomes NS, Silva SR. Avaliação da autoestima de mulheres submetidas à cirurgia oncológica mamária. Texto \& Contexto Enferm. 2013 abr-jun; 22(2):509-16.

16. Potter PA, Perry AG. Fundamentos de enfermagem. $8^{\mathrm{a}}$ ed. Rio de Janeiro: Elsevier; 2013.

17. Ministério da Saúde (BR). Instituto Nacional do Câncer. Guia de nutrição para pacientes e cuidadores: orientações aos pacientes. Rio de Janeiro: INCA; 2010.

18. Parra MV, Panobiano MS, Prado MAS, Almeida AM, Franco AHJ, Vendrusco LM. Visita domiciliar a mulheres com câncer de mama: uma estratégia a ser resgatada. Ciênc Cuid Saúde. 2010;9(2):301-8.

19. Fernandes AFC, Bonfim IM, Araújo IMA, Silva RM, Barbosa ICFJ, Santos MCL. Significado do cuidado familiar à mulher mastectomizada. Esc Anna Nery Rev Enferm. 2012 jan-mar; 16(1):27-33.

Data de recebimento: $25 / 08 / 2013$

Data de aceite: $28 / 11 / 2013$

Contato com autor responsável: Sueli Riul da Silva

Endereço postal: Rua Donaldo Silvestre Cicci, 665. Uberaba, MG, Brasil. Cep 38082-166.

E-mail sueliriul@terra.com.br 